\title{
Blackout: Understanding transient vision loss
}

Rachael C Heath Jeffery, Fred K Chen, Christian J Lueck

\section{Background \\ Transient vision loss may indicate underlying disease of the eye, optic nerve, orbit, brain or heart. Detailed history- taking followed by a complete ocular and neurological examination is therefore a crucial part of any consultation.}

\section{Objective}

It is important to determine whether a patient with transient vision loss can be reassured or requires urgent referral for further investigation. This review examines monocular and binocular transient vision loss and provides a structured approach to the examination of a patient with transient vision loss. The aim of this article is to provide clinicians with confidence when encountering these patients.

\section{Discussion}

Transient vision loss can imply serious underlying pathology; therefore, accurate history-taking and astute observation are paramount. This review discusses the differential diagnosis of monocular and binocular transient vision loss and the relevant localising features of each.
TRANSIENT VISION LOSS may be monocular or binocular. The aetiology may be localised to the eye, orbit, optic nerve, brain, neck or heart through careful history-taking and examination. ${ }^{1}$ Medical practitioners may become aware of previous episodes of transient vision loss while taking a patient's history for a seemingly unrelated problem. Alternatively, new-onset transient vision loss may be an early sign of a sight-threatening, and possibly life-threatening, emergency. ${ }^{2,3}$ This review aims to guide initial management and suggest referral pathways in both of these situations.

It may be difficult for a patient to distinguish monocular from binocular transient vision loss, and this is often reflected in the history. It is essential that the clinician distinguishes whether the presentation is for monocular or binocular vision loss. One way to do this is to ask whether the patient covered an eye while the problem was happening. An alternative is to ask whether the visual world was split (eg seeing half a face), as this almost always implies binocular visual loss.

The most common cause of monocular transient vision loss is amaurosis fugax, usually resulting from an embolic complication of an ulcerated plaque of the carotid artery. ${ }^{2}$ In contrast, the most common cause of transient binocular vision loss is migraine. ${ }^{4}$ It is crucial that a thorough history-taking includes an attempt at determining whether one or both eyes were affected as well as discovering the speed of onset, any precipitating factors, the speed of recovery and the presence of any residual symptoms. It is important to ask about associated visual and/or neurological symptoms, including haloes around lights, diplopia, headache, nausea, vomiting, photophobia or phonophobia. ${ }^{3}$ It is important to ask about previous migraines, transient ischaemic attacks or strokes, and neck trauma. ${ }^{1} \mathrm{~A}$ full neurological examination is recommended for all patients with unexplained transient vision loss. ${ }^{5}$

\section{Monocular transient vision loss}

The aetiology of transient vision loss in one eye may relate to pathology in the eye, optic nerve, brain, neck or heart. ${ }^{2}$ Within the eye, acute angle-closure glaucoma can cause transient unilateral visual phenomena which are often, but not always, associated with ocular pain and redness. These events may be precipitated by pupil dilation in the dark or, rarely, the use of dilating eye drops. Other ocular causes of transient monocular vision loss include incipient retinal vascular occlusion, retinal vasospasm and tear film instability. Red flags and appropriate referral pathways for transient visual loss are discussed in Table 1 . Any form of orbital tumour that compresses the optic nerve can also cause transient monocular loss, and the visual loss can sometimes be gaze-evoked in this setting. Systemic vascular disease (including giant cell 
arteritis) as well as a pituitary tumour compressing one optic nerve should both be considered in the differential diagnosis. In these cases, a relative afferent pupillary defect (RAPD) can generally be elicited. ${ }^{5}$ An RAPD is detected using the swinging torch test, in which the pupils are alternately illuminated by a light source while the patient gazes at a distant object to eliminate accommodation. If input to the brain from both eyes is equal, irrespective of whether they are normal, pupillary constriction will be the same whichever eye is illuminated. However, if there is unilateral or asymmetrical damage to the optic nerve, both pupils will be larger when the light is shone in the eye with more optic nerve damage, in comparison to when it is shone in the contralateral eye. The direct and consensual responses keep the two pupils the same size as each other, but the overall response of both pupils allows comparison of the input from the two eyes. Therefore, this test can only detect a relative dysfunction in the optic nerve and does not exclude symmetrical bilateral pathology.

Amaurosis fugax is one of the most serious causes and results from an embolus affecting the retinal circulation, usually arising from a carotid artery atherosclerotic plaque or, rarely, from cardiac valvular disease, the aorta or an internal carotid dissection. ${ }^{6}$ Assessment for atrial fibrillation is essential, and electrocardiography (ECG) and a Holter monitor test should be performed, along with assessment of the carotids by Doppler ultrasonography.

Patients may misinterpret binocular homonymous hemianopic field loss as visual loss in one eye. It is sometimes possible to clarify this on history by asking the patient whether they covered each eye in turn during the attack. Many patients do not think to do this, and an alternative strategy is to ask them whether, during the attack, the world was split so that they

\section{Table 1. Red flags for transient vision loss}

Monocular transient vision loss

\begin{tabular}{|c|c|c|}
\hline Associated clinical findings & Likely diagnosis & Referral \\
\hline $\begin{array}{l}\text { Jaw claudication and temporal scalp } \\
\text { tenderness }\end{array}$ & Giant cell arteritis & $\begin{array}{l}\text { Immediate (<24 hours) ophthalmology or } \\
\text { emergency department assessment }\end{array}$ \\
\hline $\begin{array}{l}\text { Only present when looking in a certain } \\
\text { direction of gaze }\end{array}$ & Tumour compressing the optic nerve & $\begin{array}{l}\text { Urgent ( }<24 \text { hours) imaging (computed } \\
\text { tomography of the brain and orbits), followed, } \\
\text { if appropriate, by urgent ( }<48 \text { hours) } \\
\text { neurosurgical review }\end{array}$ \\
\hline Precipitated by postural changes & Papilloedema & $\begin{array}{l}\text { Urgent ( }<24 \text { hours) imaging (computed } \\
\text { tomography of the brain and orbits) followed, } \\
\text { if appropriate, by urgent ( }<24 \text { hours) neuro- } \\
\text { ophthalmology, neurology or neurosurgical review }\end{array}$ \\
\hline Red, painful eye & Acute angle-closure glaucoma & Immediate (<1-2 hours) ophthalmology review \\
\hline $\begin{array}{l}\text { Signs or history of neck trauma }+/- \\
\text { Horner's syndrome }\end{array}$ & Dissection of internal carotid artery & Immediate emergency department assessment \\
\hline
\end{tabular}

Binocular transient vision loss

\begin{tabular}{|c|c|c|}
\hline Associated clinical findings & Likely diagnosis & Referral \\
\hline
\end{tabular}

may be associated with nausea, vomiting,

phonophobia, photophobia

Blurred vision presenting after headache onset

Valsalva manoeuvre
Occipital tumour or arteriovenous malformation
Urgent (<24 hours) imaging (computed tomography of the brain and/or angiogram) and, if appropriate, urgent ( $<24$ hours) neurosurgical review

Urgent $(<24$ hours) imaging (computed tomography of the brain and orbits) followed, if appropriate, by urgent ( $<24$ hours) neuroophthalmology, neurology or neurosurgical review

Additional neurological symptoms including vertigo, perioral numbness, gait disturbance, dysphagia and unilateral or bilateral weakness or paraesthesia
Vertebrobasilar insufficiency

Urgent (<24 hours) neurology review 
saw half a face or half a television. If the answer to this is 'yes', this implies that the problem must have occurred behind the optic chiasm.

\section{Binocular transient vision loss}

Transient vision loss in both eyes may be localised to both eyes or to both optic nerves, as in obscurations in the context of papilloedema or bilateral optic nerve compression. ${ }^{3}$ However, the aforementioned causes are less common than dysfunction of the occipital lobe(s) as a result of pathology in the brain, neck or heart. ${ }^{1,3}$ A more commonly encountered cause of bilateral visual disturbance is the visual aura of migraine, with or without headache. A key feature of this is the accompaniment of positive visual phenomenon, described as scintillations, zigzag lines, kaleidoscope effects, geometric figures, shimmering or disco lights. With regard to the neck and heart, systemic vascular disease including an embolism in the vertebrobasilar arterial system from atherosclerosis or a dissecting aneurysm can lead to transient homonymous or global vision loss. ${ }^{1}$

Vertebrobasilar disease is most common among those $>60$ years of age, with transient binocular vision loss lasting for seconds and returning to normal within seconds to minutes. These patients typically present with blurred vision or complete loss of vision in both eyes. Bilateral global vision loss (loss of vision in both eyes) is attributed to loss of blood flow to both occipital lobes. Conversely, hemianopic loss results from transient ischaemia to only one occipital lobe. The vertebrobasilar arteries supply the brainstem, cerebellum and occipital cortex. Consequently, insufficient blood flow through this arterial system may lead to additional symptoms indicating brainstem or cerebellar involvement, such as temporary difficulty with walking or talking.

It is critical that the clinician considers transient global cerebral hypoperfusion due to orthostatic hypotension.

Conversely, progressive blackening of the patient's visual field over seconds to minutes is associated with presyncope/ syncope. This results in occipital lobe hypoperfusion, something that may be postural/hypotensive, vasovagal, cardiogenic (structural or arrythmia) or neutrally mediated. ${ }^{3}$ ECG, and possibly a Holter monitor test, forms an important part of the assessment.

\section{Examination checklist}

The first step is to observe the patient and check for the presence of proptosis, ocular redness or oedema. If the patient is older than 50 years of age, the temporal arteries should be palpated and the patient's blood pressure noted. $^{7}$

Best corrected visual acuity, pupil size, extraocular motility, visual fields to confrontation and colour vision should then be assessed. The presence of ptosis and/or extraocular muscle involvement suggests an associated cranial nerve palsy. Ptosis may also signify a carotid artery dissection. It is important to observe both the direct pupillary response in the illuminated eye and the consensual response in the fellow eye. The swinging torch test (for an RAPD) is then performed, ideally in a darkened room with a very bright light source..$^{5}$ If an RAPD is present, the response of both pupils will be diminished when the light is shone in one eye when compared with the other. ${ }^{5}$

It is recommended that the clinician auscultates the patient's neck for the presence of a carotid bruit as well as over the orbit for an intracranial fistula or aneurysm. Finally, a full neurological examination should be performed to look for evidence of pathology separate from visual pathways. ${ }^{7}$

\section{Diagnostic criteria}

Amaurosis fugax, or transient monocular vision loss, has an incidence of $14 / 100,000$ people per year. ${ }^{8,9}$ Common causes of amaurosis fugax include emboli from atherosclerotic carotid arteries, valvular heart disease or, less commonly, carotid artery dissection (Table 2)..$^{2,7}$ The patient's past medical history will typically include vasculopathic risk factors. ${ }^{7}$ Visual loss is usually sudden, with recovery occurring within one hour (typically $<10$ minutes). The patient will often describe a curtain coming down over one eye. Importantly, the unaffected eye should have maintained normal vision throughout the episode. These patients almost always have a normal ocular and neurological examination. ${ }^{10}$ All cases of amaurosis fugax warrant urgent ophthalmology or neurology referral. ${ }^{7,11}$ Studies show that patients with transient monocular vision loss associated with carotid artery atherosclerosis have a $2 \%$ risk of stroke over the next year. ${ }^{2}$ In patients with severe stenosis, the risk of ipsilateral stroke is approximately $16 \%$ over the next three years. ${ }^{2}$ Investigation and treatment must focus on secondary prevention measures to decrease the likelihood of subsequent ischaemic events. ${ }^{12}$

All cases of suspected internal carotid dissection require urgent referral to the local emergency department for magnetic resonance imaging plus magnetic resonance angiography or computed tomography plus computed tomography angiography of the neck vessels. ${ }^{12,13}$ If a dissection can be safely ruled out, the patient will then require ECG and echocardiography. ${ }^{12,14}$ Red flags to be aware of when assessing a patient with transient vision loss are listed in Table 1.

Incipient retinal artery or vein occlusions can present with transient vision loss. ${ }^{2}$ Red flags include arterial narrowing, visible cholesterol emboli and/or retinal haemorrhages as seen on direct ophthalmoscopy. ${ }^{1}$ In these cases, it is imperative to manage any factors that may prevent an impending occlusion (eg hypertension) and to consider commencing aspirin. ${ }^{15}$

Another cause of monocular vision loss localised to the eye is acute angle-closure glaucoma. ${ }^{3}$ Patients with acute angle-closure glaucoma may report pain, redness and haloes around lights in the affected eye. If the equipment is available, it is recommended that the clinician check the patient's intraocular pressure; if not, the patient should be referred for an urgent ophthalmology assessment. ${ }^{16}$

Mild unilateral vision loss may also result from dry eyes and tear film instability. Patients with this condition may complain of ocular dryness, reflex tearing or a foreign body sensation. Vision 
typically improves on blinking or with the use of lubricating eye drops.

Papilloedema can present with transient vision loss in one or both eyes. ${ }^{2}$ The vision loss ('obscuration') is typically more a 'greyout' or 'brownout' than the 'blackout' of amaurosis fugax. It typically lasts for only a few seconds and is precipitated by bending over, coughing or straining. In contrast, vision loss associated with orthostatic hypotension is usually bilateral and is precipitated by standing upright from a supine position. Similarly, optic nerve, orbital or pituitary tumours are all rare causes of transient monocular vision loss. ${ }^{17}$ Patients with these tumours may occasionally report positive visual phenomena including flashes. Transient vision loss that only occurs in a particular direction of gaze is a red flag for an orbital tumour compressing on the optic nerve (Table 1) and warrants urgent imaging and neurosurgical opinion. ${ }^{1}$

In patients aged $>50$ years, it is important to consider giant cell arteritis as a cause of transient vision loss. Pertinent history includes jaw claudication, scalp tenderness, muscle pain and fever of unknown origin. Palpation of the temporal arteries may reveal a thickened, tender and non-pulsatile vessel. Urgent referral to an emergency department is required to commence high-dose steroid treatment to prevent permanent vision loss in the affected eye and possible sequential involvement of the other eye. ${ }^{18}$ Important investigations include an urgent full blood evaluation, erythrocyte sedimentation rate, $\mathrm{C}$-reactive protein and temporal artery biopsy. ${ }^{1}$

Transient binocular visual disturbance is most commonly attributed to migraine or orthostatic hypotension in adolescents and young adults. Ideally, the patient should have a known history of true migraines or motion sickness with an onset prior to the age of 40 years. Typically, an expanding, scintillating scotoma will be reported in both eyes, usually beginning as a small area of obscuration that progressively expands over 20-30 minutes to loss of a full hemifield. ${ }^{1}$ The scotoma may be accompanied by zigzags and/or flashing lights or wavy vision. Any associated headache usually occurs after the onset of the visual phenomena, but not all migrainous visual loss is associated with headache (acephalgic migraine). A headache occurring prior to the visual changes is a red flag for a possible space-occupying lesion. ${ }^{1}$ Migrainous vision loss generally returns to normal in both eyes and may be followed by a temporary period of nausea, vomiting, photophobia, phonophobia or malaise. Patients with migrainous vision loss usually have a normal ocular and neurological examination, and there should be no evidence of features suggesting giant cell arteritis. The presence of other, or persisting, neurological symptoms warrants referral to a neurologist and may indicate a more serious cause. The Framingham study found that transient visual phenomena, similar to the visual aura of migraine, were not associated with an increased risk of stroke. ${ }^{19}$

It is important not to confuse vasospastic monocular transient vision with migraine. Patients with vasospastic monocular transient vision are typically young and are otherwise well or may have a history of Raynaud's phenomenon. The same eye is affected each time, and the episode presents with patchy, faded vision lasting approximately one

\section{Table 2. Common causes of amaurosis fugax and vertebrobasilar insufficiency}

\begin{tabular}{l}
$\begin{array}{l}\text { Amaurosis fugax } \\
\text { (monocular vision loss) }\end{array}$ \\
\hline - Internal carotid artery atherosclerosis \\
- Internal carotid artery dissection \\
- Giant cell arteritis \\
- Cardiac disease (including valvular \\
disease or patent foramen ovale)
\end{tabular}

Vertebrobasilar insufficiency (binocular vision loss)

- Vertebrobasilar arterial stenosis

- Emboli from atherosclerotic plaque

- Arterial dissection

- Giant cell arteritis minute followed by temporary poor visual acuity. Importantly, there are no positive phenomena or associated neurological symptoms. ${ }^{1}$ These patients warrant non-urgent referral to a neuro-ophthalmologist. The use of nifedipine has been shown to reduce the frequency of vasospasm. ${ }^{20}$

Vertebrobasilar insufficiency may present with sudden-onset bilateral vision loss that completely resolves within one hour, often within a few minutes. ${ }^{1}$ The vision loss may be hemianopic or global depending on whether one or both occipital lobes are affected. ${ }^{1}$ The patient is usually aged $>50$ years and, as with amaurosis fugax, is likely to have vasculopathic risk factors including ischaemic heart disease, hypertension and diabetes. ${ }^{13}$ The episode of vision loss may be associated with other neurological symptoms including vertigo, perioral numbness, gait disturbance, dysphagia, unilateral or bilateral weakness, or paraesthesiae. ${ }^{1}$ These patients require referral for a full neurological exam, cardiac review and evaluation of postural blood pressure changes.

\section{Conclusion}

There are many possible causes of monocular and binocular transient vision loss. Importantly, transient monocular vision loss is considered the retinal equivalent of a cerebral transient ischaemic attack. The clinician must determine whether a patient with transient vision loss can be reassured or, instead, requires urgent referral to the local emergency department for further investigation. If any uncertainty exists, onward referral is appropriate granted the potential for serious underlying systemic pathology.

\section{Authors}

Rachael C Heath Jeffery MChD, MPH, Medical Retina Fellow, Centre for Ophthalmology and Visual Science (incorporating Lions Eye Institute), The University of Western Australia, WA; Associate Clinical Lecturer Australian National University Medical School, Canberra Hospital, ACT

Fred K Chen PhD, FRANZCO, Senior Lecturer, Centre for Ophthalmology and Visual Science (incorporating Lions Eye Institute), The University of Western Australia, WA; Ophthalmology Consultant, Vitreoretinal Surgery, Department of Ophthalmology, 
Royal Perth Hospital, WA; Ophthalmology Consultant, Department of Ophthalmology, Perth Children's Hospital, WA

Christian J Lueck PhD, FRACP, FRCP(UK), FAAN, Professor, Australian National University Medical School, Canberra Hospital, ACT; Senior Staff Specialist and Head, Department of Neurology, Canberra Hospital, ACT

Competing interests: None.

Funding: None.

Provenance and peer review: Not commissioned, externally peer reviewed.

\section{Correspondence to:}

rachaelheathjeffery@lei.org.au

\section{References}

1. Miller NR, Newman NJ. Walsh and Hoyt's clinical neuro-ophthalmology. 6th edn. Philadelphia, PA: Lippincott Williams and Wilkins, 2005.

2. Volkers EJ, Donders RC, Koudstaal PJ, van Gijn J, Algra A, Jaap Kappelle L. Transient monocular blindness and the risk of vascular complications according to subtype: A prospective cohort study. J Neurol 2016;263(9):1771-77. doi: 10.1007/s00415016-8189-x.

3. Pula JH, Kwan K, Yuen CA, Kattah JC. Update on the evaluation of transient vision loss. Clin Ophthalmol 2016;10:297-303. doi: 10.2147/OPTH. S94971.

4. Burde RM, Savino PJ, Trobe JD. Clinical decisions in neuro-ophthalmology. 3rd edn. St Louis, MO: Mosby, 2002.

5. Broadway DC. How to test for a relative afferent pupillary defect (RAPD). Community Eye Health 2012;25(79-80):58-59.

6. Ahmed R, Foroozan R. Transient monocular visual loss. Neurol Clin 2010;28(3):619-29. doi: 10.1016/j. ncl.2010.03.004.

7. Bidot S, Biotti D. Transient monocular blindness: Vascular causes and differential diagnoses. J Fr Ophtalmol 2018;41(4):e129-e36. doi: 10.1016/j. jfo.2017.12.002.

8. Vodopivec I, Rizzo JF 3rd. Ophthalmic manifestations of giant cell arteritis. Rheumatology 2018;57(suppl_2):ii63-ii72. doi: 10.1093/rheumatology/kex428.

9. Mokri B. Traumatic and spontaneous extracranial internal carotid artery dissections. J Neurol 1990;237(6):356-61. doi: 10.1007/BF00315659.

10. Guillon B, Lévy C, Bousser MG. Internal carotid artery dissection: An update. J Neuro Sci 1998;153(2):146-58. doi: 10.1016/s0022510x(97)00287-6.

11. Sharma RA, Newman NJ, Biousse V. New concepts on acute ocular ischemia. Curr Opin Neurol 2019;32(1):19-24. doi: 10.1097/ WC0.0000000000000634.

12. Prasad S. A window to the brain: Neuroophthalmology for the primary care practitioner. Am J Med 2018;131(2):120-28. doi: 10.1016/j. amjmed.2017.10.008.

13. Dattilo M, Newman NJ, Biousse V. Acute retinal arterial ischemia. Ann Eye Sci 2018;3:28. doi: 10.21037/aes.2018.05.04.

14. Vodopivec I, Cestari DM, Rizzo JF 3rd. Management of transient monocular vision loss and retinal artery occlusions. Semin Ophthalmol 2017;32(1):125-33. doi: 10.1080/08820538.2016.1228417.

15. Lawlor M, Perry R, Hunt BJ, Plant GT. Strokes and vision: The management of ischemic arterial disease affecting the retina and occipital lobe. Surv Ophthalmol 2015;60(4):296-309. doi: 10.1016/j.survophthal.2014.12.003.
16. Andersen CU, Marquardsen J, Mikkelsen B, Nehen JH, Pedersen KK, Vesterlund T. Amaurosis fugax in a Danish community: A prospective study. Stroke 1988;19(2):196-99. doi: 10.1161/01. str.19.2.196.

17. Dasgupta B, Borg FA, Hassan N, et al. BSR and BHPR guidelines for the management of giant cell arteritis. BSR and BHPR Standards, Guidelines and Audit Working Group. Rheumatology (Oxford) 2010;49:1594-97. doi: 10.1093/rheumatology/ keq039a.

18. Bruno A, Jones WL, Austin JK, Carter S, Qualls C. Vascular outcome in men with asymptomatic retinal cholesterol emboli. A cohort study. Ann Intern Med 1995;15:122:249-53. doi: 10.7326/0003-4819-122-4-19950215000002.

19. Wijman CA, Wolf PA, Kase CS, Kelly-Hayes M, Beiser AS. Migrainous visual accompaniments are not rare in late life: The Framingham study. Stroke 1998;29(8):1539-43. doi: 10.1161/01.str.29.8.1539.

20. Pitkänen $H$, Saarela V. Vasospastic transient monocular visual loss: Effect of treatment with different doses of nifedipine. J Neuroophthalmol 2014;34(4):386-88. doi: 10.1097/ WNO.0000000000000144. 